\title{
A REVIEW ON PHYTOSOMES, THEIR CHARACTERIZATION, ADVANCEMENT \& POTENTIAL FOR TRANSDERMAL APPLICATION
}

\author{
${ }^{*}$ Surendra Tripathy ${ }^{\mathrm{a}}$, Dilip K Patel ${ }^{\mathrm{a}}$, Lipika Baro ${ }^{\mathrm{b}}$, Suresh K Nair ${ }^{\mathrm{a}}$ \\ ${ }^{a}$ Chandra Shekhar Singh College Of Pharmacy, Koilaha, Kaushambi, Allahabad, India 212213. \\ ${ }^{\mathrm{b}}$ Department of Pharmaceutical Sciences, Dibrugarh University, Assam, India 786004. \\ *Corresponding Author's e-mail: surendratripathy210@gmail.com, Contact: + 91-9554870513
}

\begin{abstract}
The objective of this review is to focus on the application of phytosome technology along with its preparation, various properties and characterization. The recent development and conducted works of various researchers have been studied thoroughly to establish the transdermal route as a potential way to deliver phytoconstituents. Plant derived products or plant extracts are increasingly receiving attention as dietary supplements for the homeostatic management of inflammation, toxicities, cancers, weight loss and other chronic or acute degenerative disorders. But these products frequently face stability and bioavailability problems. Plant products after their isolation become prone to instability and are potentially unfit to cross the biomembra ne as such. Some plant products show hydrophobicity and their delivery to systemic circulation is a quite difficult task. The phytosome technique reduces these tasks to reasonable extents. Phytosomes or herbosomes are lipid compatible molecular complexes produced by interaction of standardized plant extracts and phospholipids with a stoichiometric ratio, having better stability, pharmacokinetics and pharmacodynamics profile. The phytosome or Herbosome technique increases the hydrophilicity of highly lipophilic drug there by making it suitable for drug delivery and increases the lipophilicity of hydrophilic phytoconstituents adequately to cross biological membrane. The topical application of phytosomes for cosmetic purpose has already been proven. This review contains a comparative account of liposomes and phytosomes along with recent advancements in the field of phytosome technology with a special concern to transdermal drug delivery.
\end{abstract}

Key words: Phytosomes, phospholipids, bioavailability, topical route, transdermal drug delivery

\section{INTRODUCTION}

Plants are endowed with health promoting substances which are mainly the secondary metabolites like flavonoids. Most of the biologically active constituents of plants are polar or water soluble molecules. Toxicity and absorption problem limit the use of these constituents. Apart from that the herbal extracts are destroyed by the digestive secretions and gut bacteria. Extensive researches have been conducted for successful delivery of these plant derived products since the last century ${ }^{1}$.

The term "phyto" means plant and "some" means cell like $^{2 .}$ It is also mentioned as herbosomes. This is a new patented technology, where standardized plant extracts or water soluble phytoconstituents are complexed with phospholipids to produce lipid compatible molecular complexes, there by greatly increasing absorption and bioavailability ${ }^{3,4}$. Phosphatidylcholine, phosphatidylserine, phosphatidylethanolamine, phosphatidylinositol are the phospholipids used, but phosphatidylcholine are widely used because of their certain therapeutic value in case of liver diseases, alcoholic steatosis, drug induced liver damage and hepatitis. Phospholipids are also employed as natural digestive aids and as carriers for both fat miscible and water miscible nutrients ${ }^{5}$. Phytosomes can easily traverse the lipophilic path of the enterohepatic cell membranes ${ }^{6}$ and also stratum corneum layer of the $\operatorname{skin}^{3,7-}$ 11 .

Standardized plant extracts mainly flavonoids are derived as phytosomes. Selection of flavonoids are done from the groups consisting of quercetin, kaemferol, quercretin-3, rhamnoglucoside, quercetin-3-rhamnoside, hyperoside, vitexine, diosmine, 3-rhamnoside, (+) catechin, (-) epicatechin, apigenin-7-glucoside, luteolin, luteolinglucoside, ginkgonetine, isoginkgonetine and bilobetine etc.

\section{PHYTOSOME TECHNOLOGY \& ITS ADVANTAGE}

The phytosome technology was developed by Indena s.p.A of Italy, markedly enhancing the bioavailability of selected phytomedicines, by incorporating phospholipids into standardized plant extract, which improve their absorption and utilization. The polyphenols are little soluble both in water and in lipids. The polar functionalities of the lipophilic guest interact via hydrogen bonds and polar interaction with the charged phosphate head of phospholipids, forming a unique arrangement that can be evidenced by spectroscopy ${ }^{8}$. As phosphatidylcholine is a bifunctional molecule with hydrophilic choline and hydrophobic phosphatidyl group (Fig.1), the choline group head binds with the compound, while phosphatidyl portion envelopes the bounded part (Fig.2).

The first phytosome generation was prepared by combining selected polyphenolic extract with phospholipids in non-polar solvent ${ }^{3}$, but recently the phytosome generations are developed by using hydroethanolic solvent, to comply with current food specifications $^{12}$.

As compared to conventional herbal formulation, phytosome have following advantages ${ }^{13-16}$ :

- Greater therapeutic benefits, as absorption of lipid insoluble polar botanical extracts through oral and topical route is enhanced markedly. 
- Small dose is required, as absorption is increased manifolds.

- Phytosomes possess better drug entrapment efficiency.

- Phosphatidylcholine is not merely a carrier; it is also having hepatoprotective activity and nutritional value.

- Due to formation of chemical bonds, phytosomes show better stability profile.

- Phytosomes can be used for systemic targeting of herbal drugs, as phytosome can easily transit from hydrophilic environment into the lipid friendly environment of the enterocyte cell membrane and from there to into the cell.

- Cosmetic and other topical use of phytoconstituents can be done by phytosome formulations.

\section{PREPARATION METHOD OF PHYTOSOMES}

Phytosomes are prepared by reacting a synthetic or natural phospholipid with the standardized plant extract in a ratio ranging from 0.5-2.0. But usually $1: 1$ ratio is preferable. The reaction is carried out alone or in the natural mixture in aprotic solvent, such as, dioxane, methylene chloride, acetone; from which the novel complex can be isolated by precipitation with a non-solvent, usually an aliphatic hydrocarbon or by lyophilisation or by spay drying. The solubilization or complex formation is sometimes carried out by refluxing the stoichiometric ratio mixture for a specified period in the aprotic solvent.

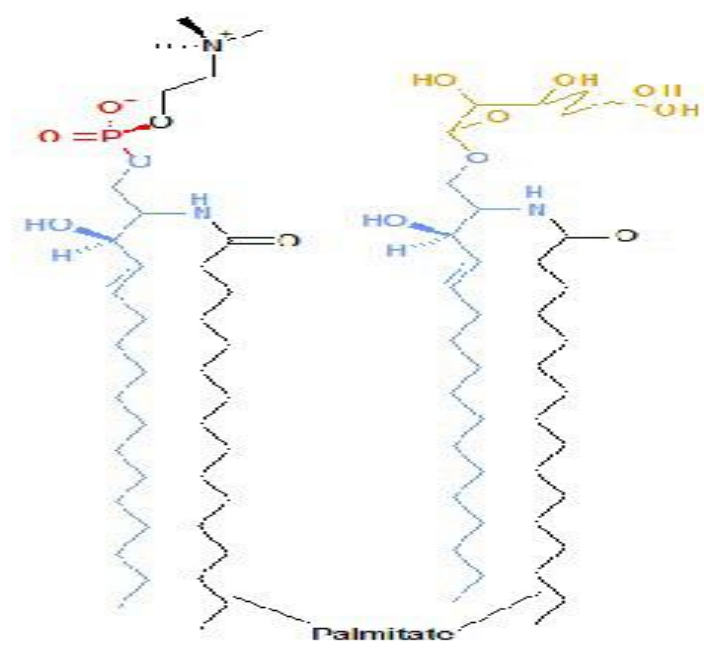

Figure1: Structure of Phosphatidylcholine

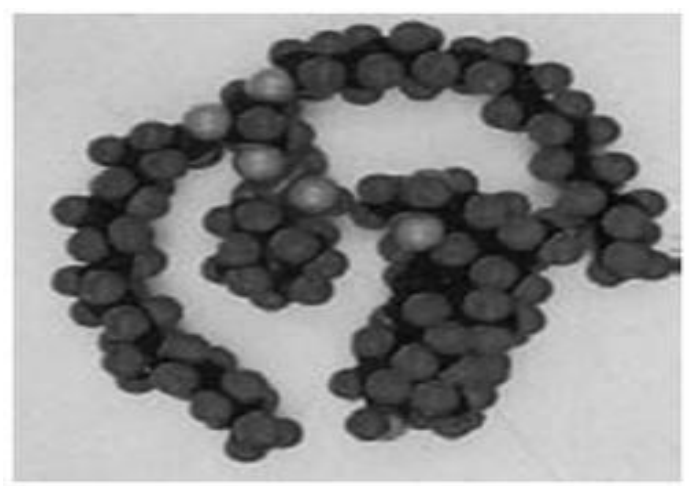

Figure 2: Molecular arrangement showing hydrophobic tail surrounding the hydrophilic choline part
The solvents may be evaporated by using rotary evaporators. Hence, after drying, the resulting complexes are soluble in polar and aprotic solvents, in which the individual components of the complex are usually insoluble. For the preparation of phytosome the phospholipids are choosen from the group consisting of soylecithin, from bovine or swine brain or dermis, phosphatidylcholine, phosphatidylethanolamine, phosphatidylserine, in which acyl group may be same or different and mostly derived from palmitic, stearic, oleic and linoleic acid ${ }^{17-21}$.

\section{PROPERTIES OF PHYTOSOMES}

\section{Physico-chemical properties}

- As previously discussed, phytosomes are prepared by reaction of stoichiometric amount of phospholipid with the standardized plant extract as substrate. The spectroscopic data reveals that the phopspholipid- substrate interaction is due to the formation of hydrogen bond between the polar head (i.e., phosphate and ammonium group) and the polar functionalities of the substrate ${ }^{22}$.

- $\quad$ The size of phytosome varies from $50 \mathrm{~nm}$ to a few hundred $\mu \mathrm{m}$.

- $\quad$ Phytosomes when treated with water, they assume a micellar shape resembling liposome and Photon Correlation Spectroscopy (PCS) reveals these liposomal structures acquired by Phytosomes.

- $\quad$ From the 1HNMR and 13CNMR data, it can be deduced that the fatty chain gives unchanged signals both in free phospholipid and in the complex, which indicates that long aliphatic chains are wrapped around the active principle, producing lipophilic envelope.

- $\quad$ Regarding the solubility of phytosomes, the complexes are often freely soluble in aprotic solvents, moderately soluble in fats, insoluble in water and relatively unstable in alcohol. But the phytosomes of certain lipophilic phytoconstituents like curcumin has shown increased water solubility upon complexation with phospholipids which has been discussed later in this paper.

\section{Biological properties}

Phytosomes are novel complexes which are better absorbed and utilized, hence they produce more bioavailability and better result than the conventional herbal extract or non-complexed extracts, which has been demonstrated by pharmacokinetic studies or by pharmacodynamic tests in experimental animals and in human subjects ${ }^{23}$.

Phytosomes express their behaviour in physical or biological system because of their physical size, membrane permeability, percentage entrapment, chemical composition, quantity and purity of the materials used. The phytosomes should not be confused with liposomes where hydrophilic drug molecules are entrapped within a cavity or spaces between the membranes. The liposomes may involve several hundred phospholipid molecules for this entrapment and are usually now being used for cosmetic purposes. Instead, the phytosomes involves interaction of 
1- 4 phospholipid molecules with the phytoconstituents which are chemically anchored to each other. Several researches have shown the phytosomes to be a better alternative for liposomes in terms of membrane permeability and stability. Fig.3 illustrates a comparative account of phytosomes with liposomes.

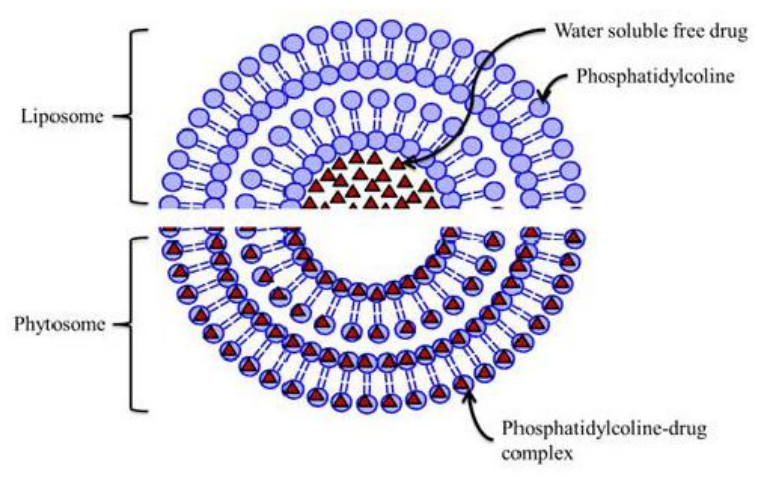

Figure 3: Structural comparision of liposome with phytosome

\section{CHARACTERIZATION OF PHYTOSOMES}

The physical attributes like shape, size, distribution, drug entrapment capacity, drug release and chemical composition are used for their characterization. Methods used for their characterization are Melting point determination, Thin Layer Chromatography (TLC), Infra Red Spectroscopy, NMR spectroscopy, Differential Scanning Calorimetry, X-Ray Diffraction Analysis, Scanning Electron Microscopy (SEM), Transmission Electron Microscopy (TEM), Photon correlation Spectroscpy (PCS), Percentage drug entrapment etc ${ }^{24}$.

The melting point of the prepared phytosomes significantly vary from that of the phytoconstituents and the phospholipid, which is due to the chemical interaction between the both moiety and the change in shape of the phytosome. The HP-TLC is a simple method used for characterization of Phytosome. The phytosomes have a retention factor value different from the phytoconstituents and phospholipids when they are eluted with the suitable solvent system ${ }^{18}$, which confirms the formation of a new molecular entity.

The instrumental techniques used for the evaluation provide major informations. The FT-IR spectra of the phytoconstituent, the phospholipid and their phytosomes are compared. The free hydroxyl group interact with the choline part of phospholipid. The peak corresponding to the free hydroxyl group changes and a broad peak appears instead. The chemical shift value, presence and absence of NMR peak of particular proton can be investigated to characterize phytosome. The H-NMR Spectra of phytosomes of various phytoconstituents show the peak of long tail part of phospholipid molecule intact, which reveals that the tail part does not take part in any chemical interactions and behaves as a sheath to the central choline part attached to phytoconstituent ${ }^{25}$. The ${ }^{13} \mathrm{C}-\mathrm{NMR}$ is often performed for confirmation of the type of interaction involved for the complexation.
The crystallinity of the phytoconstituents is lost upon complexation, which is responsible for increasing the hydrophilicity of hydrophobic phytoconstituent and balancing the hydrophilicity and lipophilicity. The crystallinity along with the interaction of phospholipid with phytoconstituent can be confirmed by the DSC and $\mathrm{X}-\mathrm{RD}$ Analysis. The crystalline drug moiety shows a sharp peak at high melting point in DSC thermogram. The phytosome show a broad peak and a melting point significantly less than that of pure drug. The broad peak indicates the loss of crystallinity. Similarly the diffraction angle $(2 \theta)$ of phospholipid, phytoconstituent and the phytosomes are compared. The general XRD study indicates loss of crystalline peaks of drugs which confirms the interaction and entrapment of drug within a sheath ${ }^{26}$.

The study of surface morphology is often important in identifying the entrapment behaviour, surface properties along with the presence or absence of impurities on the surface. The SEM provides photomicrograph of the phytosomes at suitable magnification after coating it with a very thin layer of gold. The surface of phytosomes generally shows no crystalline particles or any impurity on surface. The spherical bulging on the surface are generally obtained, which confirms the spherical shape of the phytosomes (Fig.4). The internal environment where the drug is entrapped and its distribution within the phospholipid mesh may be clearly investigated from the TEM study ${ }^{27,24}$ (Fig.5).

- The Photon Correlation Spectroscopy (PCS) technique is used for investigating the size of the phytosome and for confirmation of the vesicular structure after hydration and determination of size of vesicle. The polydispersity of vesicles are observed by this technique which gives an idea about distribution of various sizes of vesicular structures. The PCS study generally shows that the phytosomes upon hydration mostly forms unilamellar liposomal structures.

The percentage drug entrapment can be determined by extracting the phytosomes with suitable solvent systems by centrifugation at higher rpm value for short time or a lower rpm value for longer period and estimating of the supernatant for detection of drug either by UV-Visible spectroscopy or High Performance Liquid Chrromatography (HPLC) method.

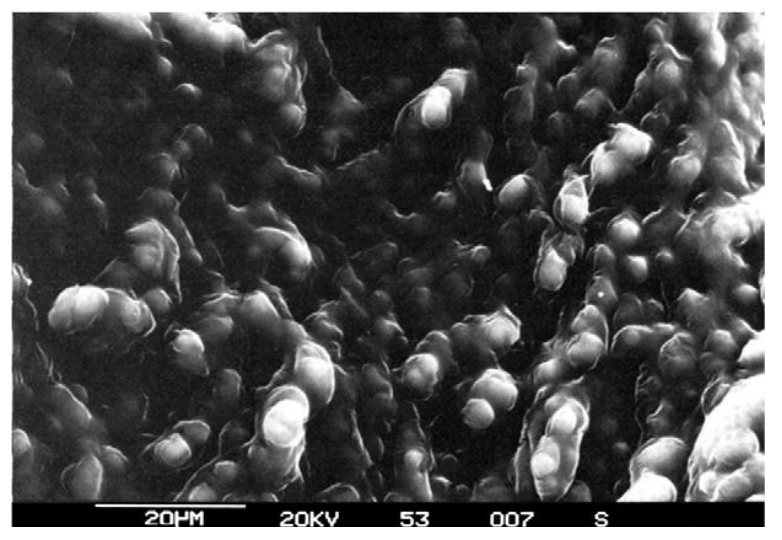

Figure 4: SEM photomicrograph of Phytosomes ${ }^{27}$ 


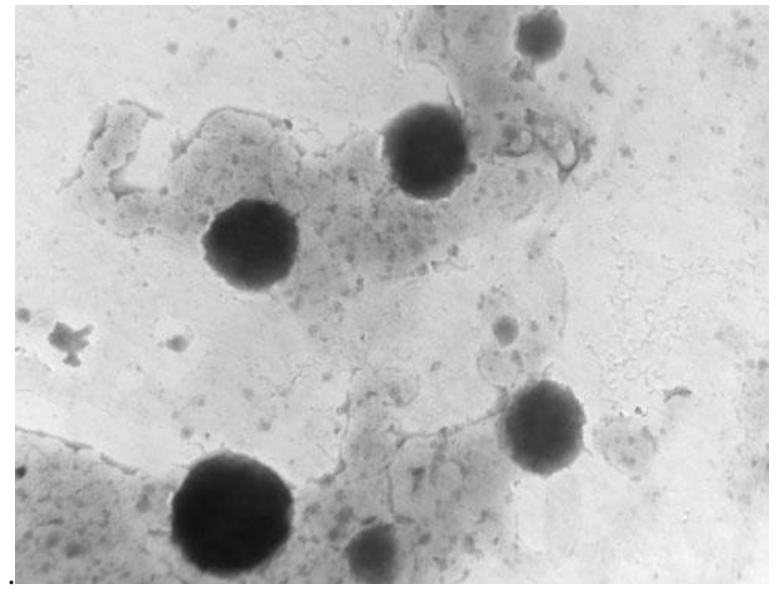

Figure 5: TEM photograph of phytosomes ${ }^{24}$

\section{ADVANCEMENT \& RECENT RESEARCHES ON PHYTOSOMES}

Numerous research is being conducted by the researchers and the recent researches reveals that the phytosome technology is a novel method for improving the absorption and bioavailability of plant extracts significantly reducing the dose level. Some plant extracts are getting more focus now-a-days due to their potential pharmacological effects, such as, silymarin, grape seed extract, quercetin, curcumin, hesperetin, ginkgo biloba extract, andrographolide etc ${ }^{28,29}$. The suitability of this technique and increased demand of herbal medicines for various disease management in current scenario, has paved the way of newer researches. Some of the crucial works of various researchers are briefly given below.

Schandalik et al, 1992, 1994, used nine human volunteer patients and tested the hepatoprotective activity of silymarin and reported that phytosomal form of silybin possess four times greater passage through the liver ${ }^{30,31}$. Similar experiment was conducted by Mascarella et al, 1993, using 232 patients with chronic hepatitis. He also reported better bioavailability of silymarin phytosome than the uncomplexed form ${ }^{20}$.

Grange et al, 1999, conducted series of experiments on silymarin phytosomes and reported the better fetoprotectant activity of the phytosomal form ${ }^{32}$. Busby et $a l$, 2002, also reported better fetoprotectant activity of silymarin phytosome than uncomplexed silymarin against ethanol-induced behavioural deficit ${ }^{33}$.

Jhiang et al, 2001, prepared Herba Epimedii flavonoid phytosome (EPF) by means of solvent evaporation technique and the accumulative dissolution of different ratios of EPF-PVP precipitate was investigated by dissolution study. The study showed that the dissolution of the precipitate was significantly higher than that of its physical mixture and Herba Epimedii extract tablets ${ }^{34}$.

Bombardelli et al, 2005, reported that the silymarin phytosomes showed much higher specific activity and a longer lasting action than the single constituent, with respect to percent reduction of edema, inhibition of myeloperoxidase activity, antioxidant and free radical scavenging activity ${ }^{35}$.
Maiti et al, 2005, developed a quercetin phytosome by a simple and reproducible method and reported that the phytosomal complex shows better therapeutic efficacy than the uncomplexed molecule in rat liver injury induced by carbon tetrachloride ${ }^{36}$. They also recently reported the enhanced bioavailability and hepatoprotective activity of andographolide herbosome by an experiment on rats and found that the phytosomal form has an enhanced activity than that of uncomplexed andrographolide at the same dose leevel ${ }^{37}$. Maiti et al, 2006, 2007, also developed naringenin and curcumin phytosomes in two different studies and reported that antioxidant activity of the phytosomal complex has better therapeutic efficacy ${ }^{19}{ }^{18}$. They developed simple methods of preparation of phytosomes.

Xiao et al, 2006, Prepared silymarin phytosomes and studied its pharmacokinetics in rats and reported that the bioavailability of silybin has increased markedly in phytosomal formulation ${ }^{21}$. Hepatoprotective activity of silymarin phytosomes was found to be more than silymarin alone against aflatoxin B1, as reported by Tedesco et al, 2008, after performing the experiment on broiler chicks ${ }^{38}$.

Green tea extract generally contains a totally standardized polyphenolic fraction (epigallocatechin and its derivatives), having a long term beneficial activities, such as, antioxidant, anticarcinogenic, antimutagenic, antiatherosclerotic, hypocholesterolemic, cardioprotective and antibacterial effects, but having a poor oral bioavailability. But the phytosomal formulation strongly improves the oral bioavailability. Further advancement in phytosome technology has been discussed in the following section.

\section{FEASIBILITY OF PHYTOSOME FOR TRANSDERMAL APPLICATION}

The enhanced bioavailability of phytoconstituents by transdermal route is a major breakthrough in the research of transdermal drug delivery systems (TDDS). TDDS involves the delivery of therapeutic agent via skin in to the systemic circulation. The stratum corneum, which is the outermost horny layer, provides the major hindrance for drug delivery due to its lipophilicity. The phytosomal form of the phytoconstituent is helpful in avoiding this barrier property. The successful research experience of around two decades of study by different researchers has been summarized below, which indicates that the phytosome technology has enough potency to be used transdermally for increasing bioavailability of phytoconstituents.

Bombardelli et al, (1991) observed that phospholipid exhibit a marked affinity for some classes of flavonoids. They developed a new series of compounds called Phytosomes, which he obtained by complexation of phospholipid with very polar botanical derivatives, i.e. complexes between a pure phospholipid and pure active principle. He first established some chemicophysical properties of phytosomes of catechin, quercetin, escin and glycyrrhetinic acid and gathered some pharmacological data. He, from the beginning described phytosomes to be a good carrier for phytoconstituents across the $\operatorname{skin}^{8}$. 
Loggia et al, (1996) prepared Gingko biloba terpenes phytosomes and reported that the phytosome was effective on soothing individual contact reaction to other substances contained in topical formulation ${ }^{9}$.

Yanyu et al, (2006) investigated that the well known soothing activity of silymarin is increased by more than six times in sylimarin phytosome in experimental models. The improvement in the activity of the phytosome form compared to the free active principle is due to a higher affinity of the complex for skin phospholipid ${ }^{21}$.

Kidd (2009) reported the hydration of the superficial corneous layer is related to the liposomal like properties of the phospholipid of the complex. Ginselect phytosomes posses a transdermic action which helps the ginseng saponin present in the phospholipid complex to penetrate into the $\operatorname{skin}^{39}$.

Forster et al, (2009) and Chanchal and Swarnlata (2008) reported in there review that the topical delivery of plant derived products can be effectively done in cosmetic preparation by phospholipid complexation ${ }^{10,11}$.

Cao et al, (2010) formulated Oxymatrine-phospholipid complex (OMT-PLC) to improve the lipid solubility and effectiveness of OMT. The purpose of their study was to explore the utility of the combination of a microemulsion and an OMT-PLC as topical delivery vehicle for enhancing the absorption and efficacy of OMT. The solubility of OMT-PLC was determined and phase diagram of microemulsion were constructed. They developed various physicochemical properties and In Vitro and In Vitro permeability through skin. They concluded that the combination of a microemulsion and phospholipid complex represents an effective vehicle for topical delivery of $\mathrm{OMT}^{40}$

Gupta and Dixit, (2011) reported that incorporation of high amount of curcumin in topical formulation cannot provide a better bioavailability. They prepared complex of curcumin with phosphatidylcholine and characterized them on the basis of TLC, DSC, Melting point and
FT-IR. They compared the activity of vesicular systems like liposome, niosome, phytovesicle. In result they got that the phytovesicles are having excellent antioxidant and antiaging properties than the other vesicular systems, that may be due to the amphiphilic nature of the complex, which greatly enhances the water and lipid miscibility of the curcumin ${ }^{24}$.

Cuomo et al, (2011) investigated the relative absorption of a standardized curcuminoids mixture and its corresponding lecithin formulation (Meriva) in a randomized, double blind cross over design human study. They reported the improved absorption and a better plasma curcuminoid profile of the Meriva at a dose significantly lower than unformulated curcuminoid mixture ${ }^{41}$.

Zaveri et al, (2011) have prepared the curcuminphospholipid complex in a molar ratio of $(1: 2)$ of curcumin and phospholipid. They confirmed the formation of complex by FT-IR Spectroscopy and DSC analysis. They compared the skin permeation of curcumin with the complexed curcumin and found that the complexed curcumin showed $60 \%$ greater permeation of curcumin through rat skin. They reported that the phospholipid complex has more transdermal penetration than pure curcumin $^{42}$.

\section{CONCLUSION}

Phytosomes or herbosomes are advanced and novel form of botanicals and phytoconstituents that are better absorbed both orally, topically and transdermally. Phytosomes have improved pharmacokinetics and pharmacological parameters and having wide scope in cosmeticology. The preparation methods of phytosomes are non-conventional, simple and reproducible. Apart from that the phospholipids used have their own beneficial effect to the body. Many areas of phytosomes are to be reported in the future in the prospect of pharmaceutical application. The phytosome technology forms a link between the conventional delivery system of phytoconstituents and novel drug delivery systems.

\section{REFERENCES}

1. Middleton E, Kandaswami C, The impact of plant flavonoids on mammalian biology: implications for immunity, inflammation, and cancer. In: Harborne JB, editor, The Flavonoids: Advances in Research Since 1986. $1^{\text {st }}$ Ed, 1994, London: Chapman and Hall; 1994. p. 619-652.

2. Mukherjee PK, Wahile A, Integrated Approaches towards drug development from Ayurveda and other Indian System of Medicine, Journal of Ethnopharmacology, 2006, 103,25-35.

3. Bombardelli E, Curri SB, Loggia Della R, Del NP, Tubaro A, Gariboldi P, Complexes between phospholipids and vegetal derivatives of biological interest, Fitoterapia, 1989,60,1-9.

4. Murray D, Phytosomes- Increase the absorption of herbal extract, 2011, Available at: http://doctormurray.com/phytosomes-herbalsupport-increase-the-absorption-of-herbal-extracts/, Accessed on: $18^{\text {th }}$ April, 2013

5. Pandey Shivanand, Patel Kinjal, Phytosomes:Technical Revolution in Phytomedicine, International Journal of PharmTech Research, 2010,2, (1), 627-631.

6. Kidd PM, Phosphatidylcholine: a superior protectant against liver damage, Alternative Medicine Review, 1996, 1, 258-74.

7. Bombardelli E, Phytosome: new cosmetic delivery system. Bollettino Chimico Farmaceutico, 1991,130 (11), 431-38.

(C) 2011, JDDT. All Rights Reserved

8. Bombardelli E and Spelta M, Phospholipid-polyphenol complex: A new concept in skin care ingredients, Cosmetics Toiletries, 1991, 106, 69-76.

9. Loggia RD, Sosa AT, Morazzoni P, Bombardelli E, AntiInflammatory activity of some Ginkgo biloba constituents and their Phospholipid complexes, Fitoterapia, 1996, 3, 257-273.

10. Forster M, Bolzinger MA, Fessi H, Briancon S, Topical delivery of cosmetics of drugs molecular aspects of percutaneous absorption and delivery, European Journal of Dermatology, 2009, 19(4),309-323.

11. Chanchal D, Swarnlata S, Novel approaches in herbal cosmetics, Journal of Cosmetics and Dermatology, 2008, 7(2), 89-95.

12. Franceschi F, Giori A (Indena S.p.A.). Phospholipid complexes of olive fruits or leaves extract having improved bioavailability. Patent app. WO2007118631, 2007.

13. Kidd PM, Head K, A review of the bioavailability and clinical efficacy of milk thistle Phytosome: a silybinphosphatidylcholine complex. Alternative Medicine Review, 2005, 10(3), 193-203.

14. Semalty A, Semalty M, Rawat MSM, The phyto-phospholipid complexes- phytosomes: a potential therapeutic approach for herbal hepatoprotective drug delivery. Pharmacognosy Reviews, 2007; 1 (2), 369-374. 
15. Naik SR, Panda VS, Hepatoprotective effect of Ginkgoselect Phytosome in rifampicin induced liver injury in rats: evidence of antioxidant activity, Fitoterapia, 2008, 79 (6), 439-445.

16. Bhattacharya S, Phytosomes: Emerging Strategy in Delivery of Herbal Drugs and Nutraceuticals, Pharma Times, 2009, 41, (3), 9- 12.

17. Marena C, Lampertico M, Preliminary clinical development of silipide: a new complex of silybin in toxic liver disorders, Planta Medica, 1991, 57,124-25.

18. Maiti K, Mukherjee K, Gantait A, Saha BP, Mukherjee PK, Curcumin-phospholipid complex: Preparation, therapeutic evaluation and pharmacokinetic study in rats, International Journal of Pharmaceutics, 2007, 330, 155-163.

19. Maiti K, Mukherjee K, Gantait A, Saha BP, Mukherjee PK, Enhanced therapeutic potential of naringenin-phospholipid complex in rats, Journal of Pharmacy \& Pharmacology, 2006, 58 (9), 1227-33.

20. Mascarella S, Therapeutic and antilipoperoxidant effects of silybin-phosphatidylcholine complex in chronic liver disease, Preliminary results, Current Therapeutic Research, 1993, 53 (1), 98-102.

21. Xiao Yanyu, Song Yunmei, Chen Zhipeng, Ping Qineng, The preparation of silybin-phospholipid complex and the study on its pharmacokinetics in rats, International Journal of Pharmaceutics, 2006, 307, 77-82.

22. Manach C, Scalbert A, Morand C, Polyphenols: food sources and bioavailability, The American Journal of clinical Nutrition, 2004, 79,727-47.

23. Franco PG \& Bombardelli E, Complex compounds of bioflavonoids with phospholipids, their preparation and uses and pharmaceutical and cosmetic compositions containing them, U.S. Patent No-EPO 275005, 1998

24. Gupta N K, Dixit VK, Development and evaluation of vesicular system for curcumin delivery, Archieves of Dermatological Research, 2011, 303, 89-101.

25. Sharma SK, Kumar M, Ahuja M, Hepatoprotective study of Curcumin-soya lecithin complex. Scientia Pharmaceutica, 2008, 76,761-774.

26. Yue P-F, Zhang W-J, Yuan H-L, Yang M, Zhu W-F, Cai P-L, Process optimization, Characterization and Pharmacokinetic Evaluation in Rats of Ursodeoxycholic Acid-Phospholipid Complex. AAPS Pharm Sci Tech, 2008, 9(1), 322-329.

27. Semalty A, Semalty M, Rawat, MSM \& Franceschi F, Supramolecular phospholipid-polyphenolics interaction: The PHYTOSOME® strategy to improve the bioavailability of Phytochemicals, Fitoterapia, 2010, 81, $306-314$.

28. Wellington K, Jarvis B, Silymarin: a review of its clinical properties in the management of hepatic disorder, Bio Drugs, 2001, 15, 465-89.

29. Hikino H, Kiso Y, Wagner H, Fiebig M, Antihepatotoxic actions of flavonolignans from Silybum marianum fruits, Planta Medica, $1984,50,248-250$.
30. Schandalik R, E. Perucca, Pharmacokinetics of silybin following oral administration of silipide in patients with extrahepatic biliary obstruction, Drugs under Experimental \& Clinical Research, 1994, 20, 37-42.

31. Schandalik R, Gatti G, Perucca E, Pharmacokinetics of silybin in bile following administration of silipide and silymarin in cholecystectomy patients, Arzneimittelforschung, 1992, 42, 96468.

32. La Grange L, Wang M, Watkins R, Ortiz D, Sanchez ME, Konst J, Lee C, Reyes E, Protective effects of the flavonoids mixture, silymarin, on fetal rat brain and liver, Journal of Ethnopharmacology, 1999, 65, 53-61.

33. Busby A, La Grange L, Edwards J, Kings J, The use of a silymarin/phospholipids compound as a fetoprotectant from ethanol-induced behavioural deficits, Journal of Herbal Pharmacotherapy, 2002, 2 (1),39-47.

34. Jiang YN, Yu ZP, Yan ZM, Chen JM, Studies on preparation of herba epimedii flavanoid phytosomes and their pharmaceutics, Zhongguo Zhong Yao Za Zhi, 2001, 26 (2), 105-8.

35. Bombardelli E, Spelta M, Loggia Della R, Sosa S, Tubaro A. Aging Skin: Protective effect of silymarin- Phytosome, Fitoterapia, 1991; 62(2), 115-22, Rev 2005; 10(3), 193-203.

36. Maiti K, Mukherjee K, Gantait A, Ahamed HN, Saha BP, Mukherjee PK, Enhanced therapeutic benefit of quercetinphospholipid complex in carbon tetrachloride induced acute liver injury in rats: a comparative study, Iranian Journal of Pharmacology \& Therapeutics, 2005, 4, 84-90.

37. Maiti,k., Mukherjee,k., Murugan,V., Saha,B.P. and Mukherjee,P.K, Enhancing bioavailability and hepatoprotective activity of Andrographolide from Andrographis paniculata; a well known medicinal food, through its herbosome, Journal of the science of Food and Agriculture, 2010,90, 43-51

38. Tedesco D, Steidler S, Galletti S, Tameni M, Sonzogni O, Ravarotto L, Efficacy of silymarinphospholipid complex in reducing the toxicity of aflatoxin B1 in broiler chicks, Poultry Science, 2004, 83(11), 1839-43.

39. Kidd PM, Bioavailability and activity of phytosome complexes from botanical polyphenols: The silymarin, curcumin, Green tea and Grape seed extracts, Alternative Medicines Review, 2009, 14 (3), 226-246.

40. Cao Fa-Hao, Ou Yang W, Wang Y, Yue P, Li S, A combination of a microemulsion and a phospholipid complex for topical delivery of Oxymatrine, Archieves of Pharmcal Research, 2010, 34(4), 551-562.

41. Cuomo J, Appendio G, Dern SA, Comparative absorption of a standardized Curcuminoid mixture and its lecithin formulation, Journal of Natural Products, 2011, 74, 664-669.

42. Zaveri M, Gajjar H, Kanaki N, Patel S, Preparation and evaluation of drug phospholipid complex for increasing transdermal penetration of phytoconstituents, International Journal of Institutional Pharmacy and Life sciences, 2011, 1(3), 80-93. 